2002 Annual Report Conference on Electrical Insulation and Dielectric Phenomena

\title{
Response of a Narrow Band PD Detector and Analyzer in Ageing Experiments
}

\author{
S.Senthil Kumar, Y.P. Nerkar, M.N. Narayanachar and R.S. Nema \\ Department of High Voltage Engineering, \\ Indian Institute of Science, Bangalore, INDIA
}

\begin{abstract}
Partial discharge (PD) pulses are recorded with an analyzer connected to a PD detector. The analyzer presents PD data as distributions that are used to interpret the PD phenomenon. The paper describes results of ageing experiments on oil impregnated pressboard samples subjected to PD measured with a narrow band detector and analyzer system. The main focus of the paper is on instrumentation related with narrow band detector and analyzer. A detailed response study of the narrow band detector is carried out to understand its behavior with pulses appearing with different repetition rate. The paper discusses characterization of PD distributions for meaningful interpretation.
\end{abstract}

\section{Introduction}

Precise measurement and characterization provide the basis for scientific knowledge development. Partial discharge (PD) measurement and characterization is popular for insulation condition monitoring. This is so because PD cause degradation of insulation system and a study of their behavior can give useful information regarding the state of the insulation system.

PD is measured with a PD detector which are classified as wide band and narrow band detector. While the wide band detector has resolution and sensitivity advantages, the narrow band detector finds its application for measurement in noisy environment. Other than the difference of filter characteristics between a wide band and a narrow band detector, the narrow band detector has a pulse shaping network called as envelop detector to convert the under damped oscillating response to an unipolar pulse. A simple circuit representation of the narrow band detector is shown in Figure 1. The envelop detector acts as a low pass filter capturing the variations in the oscillation.

Wide band and narrow band PD detectors are expected to quantify PD pulses identically. They do so when they are tested with PD calibrator for their ability to detect and quantify PD pulses. However in an actual experimental conditions, the behavior of PD instruments can vary resulting into instrumentdependent detection and quantification [1]. Therefore, knowledge of instrument behavior is essen-

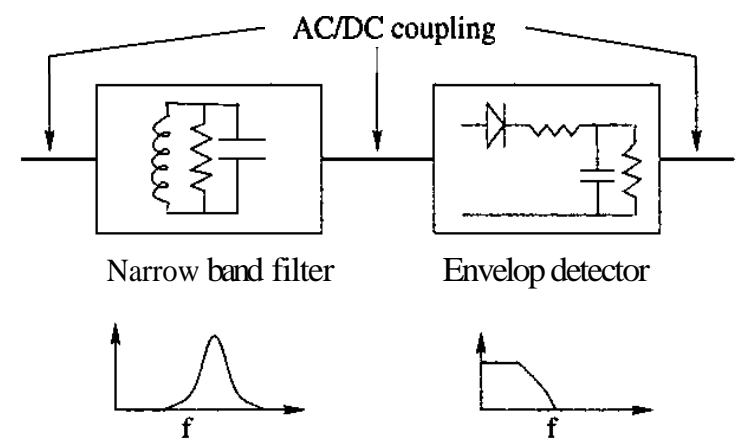

Figure 1: A simple representation of a narrow band detector

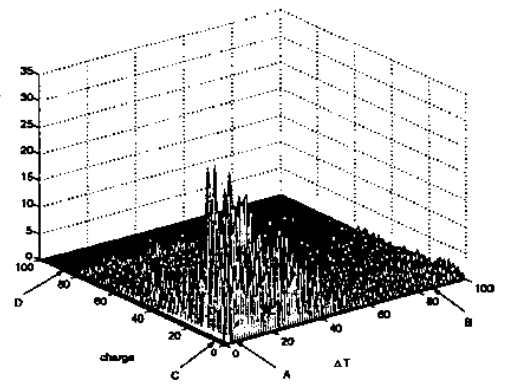

Figure 2: AT-q-n plot indicating detector dependent limits

tial for accurate PD characterization. The paper presents in two parts; (a) Response of PD Instruments and (b) Ageing Characterization, the limitations of PD instrument and its application for meaningful interpretation.

\section{Response of PD Instruments}

\section{Limits for PD measurement}

PD measurement is influenced by detector's resolution time, amplifier setting, analyzer dead time and detector-analyzer interface compatibility [1]. This makes acquisition of PD distributions instrumentdependent.

The existence of this dependence can he understood with help of Figure $\mathbf{2}$ and Figure 3 that show an uncalibrated 3D distribution of time interval and charge magnitude $(\boldsymbol{A T}-q-\boldsymbol{n})$ and $2 \mathrm{D}$ distribu- 


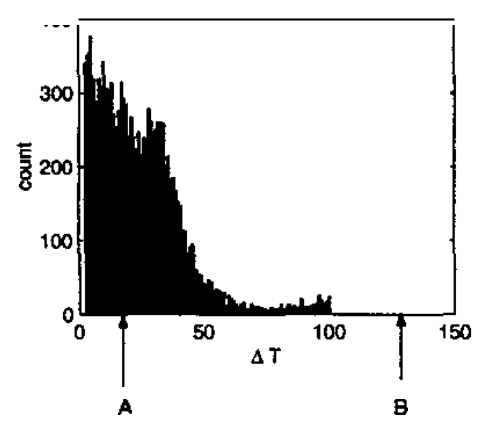

(a) $\boldsymbol{A T}$ distribution

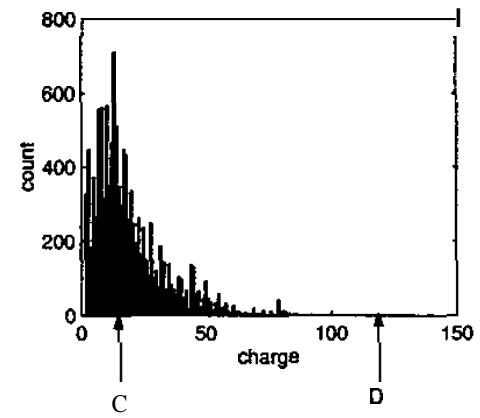

(b) Charge distribution

Figure 3: Limits appearing on measurement from a narrow band detector

tions of time interval (Figure 3(a)) and charge magnitude (Figure 3(b)) respectively. The data for the distributions is acquired using a wide band detector to conceptually demonstrate the limitations in PD measurement arising from instrument specifications.

The 3D distribution shows a presence of large number of pulses with smaller time interval and charge magnitude. The pulse counts are small for larger time interval and charge magnitude values. A narrow band detector-analyzer can record only a slice of the distribution measured by the wide band detector. Limits A-B and C-D appear on the measurement depending on the detector specifications. ' $\mathrm{A}$ ' is the limit arising due to the resolution time of the detector (or due to the dead time of the analyzer). For most cases there may not be an upper bound ' $\mathrm{B}$ ' on measurement of time interval between pulses. A bound on measurement of charge magnitude (C-D) arises depending on the sensitivity of the setup and the range supported by the detector amplifier setting. Pulse magnitude above the upper limit 'D' gets saturated and accumulates around the limit which can be easily identified from PD distributions.

Figure 4 shows a pulse appearing within the res-

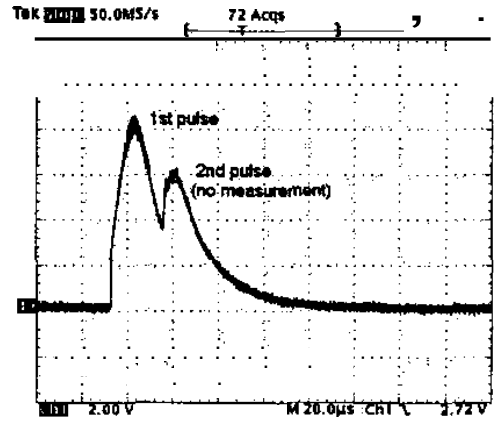

Figure 4 Pulses within the resolution time

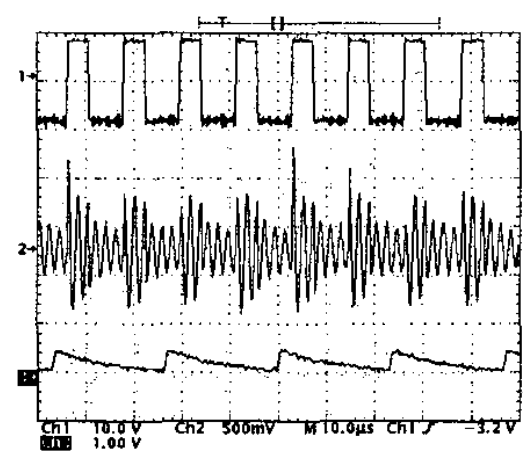

Figure 5: Response of narrow band detector for pulses with high repetition rate

olution time of the narrow band detector. The second pulse overlaps with the first and does not get recorded by the analyzer. With reference to Figure 2 that shows many small magnitude pulses having small time intervals between them, it can be expected that the narrow band detector-analyzer will not be able to respond to these pulses. The small interval pulses will suffer from resolution error and only a limited number of small magnitude pulses will get recorded. A condition for a narrow band detector where resolution error cannot be distinguished is shown in Figure 5. The oscillogram shows a square wave pulse $(\mathrm{Chl})$, the measuring impedance output (Ch2) and the narrow band detector output (Ch3). It can be clearly seen from the figure that the narrow band detector output does not show any overlapping of pulses but the measuring impedance does. The narrow band detector responds to high repetition pulses with the time constant of the envelop detector and with reduced magnitude. 

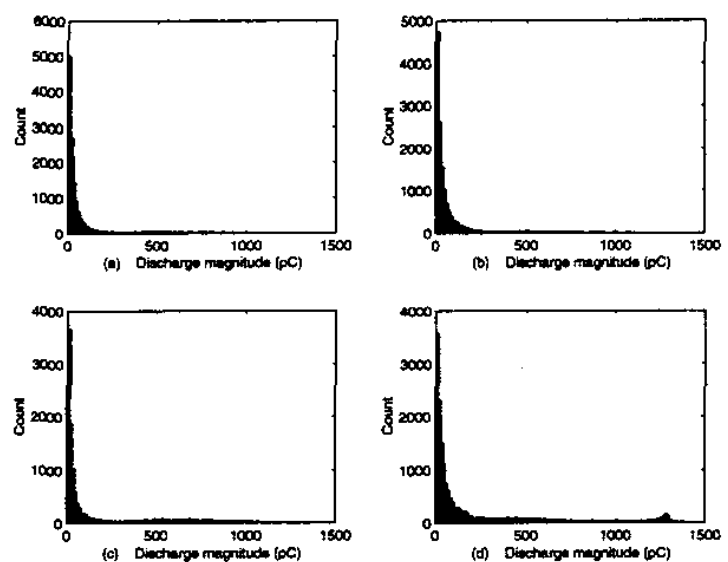

Figure 6: Charge distributions at different stages of ageing

\section{Ageing Characterization}

This section describes characterization of the results obtained from ageing experiments on model insulation consisting of oil-impregnated pressboard samples placed between $7 \pi / 12$ Rogowski electrodes. The experimental details are described elsewhere [Z, 3].

PDs occurred in the wedge shaped oil gap between the pressboard and the electrodes causing degradation of the pressboard sample. During the experiment, it was seen that the maximum charge magnitude and the number of pulses increased with time. With the experience of preliminary ageing experiments on pressboard sample, the narrow band detector ampliier setting was adjusted to $1400 \mathrm{pC}$ range [2]. In this range the charge magnitude saturated towards the end of the experiment. This range was considered optimal because at the next level of range setting the sensitivity of $\mathrm{PD}$ setup was very poor resulting in fewer PD pulse measurement compared to $1400 \mathrm{pC}$ range. PD pulses were measured with the narrow band detector-analyzer system and also were observed on a digital storage oscilloscope (DSO).

Figure 6 gives PD distributions acquired at different stages of ageing from inception to the failure of the sample. Distributions are plotted from PD records formed by $\mathbf{1 0}$ second data. Stage(a), Stage(b), Stage(c) and Stage(d) correspond to PD distribution data acquired at $25 \%, 50 \%, 75 \%$ of time to failure and just before the failure of the pressboard sample respectively.

The distributions show a large number of small

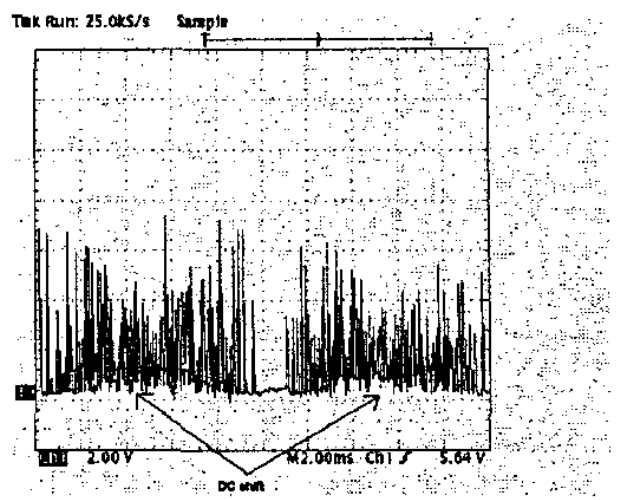

Figure 7: Oscillogram showing PD pulses for one AC cycle

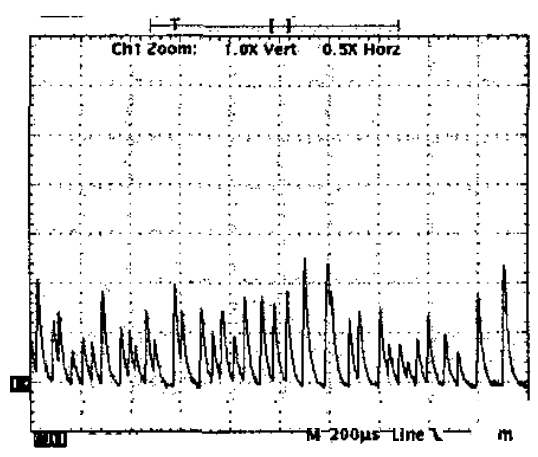

Figure 8: Many overlapping PD pulses

magnitude pulses. The pulse count of the small magnitude pulses decreases with ageing time. This $\mathrm{rep}$ resentation of small magnitude pulse count is inconsistent with observations on the oscilloscope which show increase in the number of pulses with time [2]. Figure 7 shows an oscillogram obtained for one AC cycle during the course of experiment with DC shift due to overlapping of pulses. Another oscillogram (Figure 8) was obtained for a part of the AC cycle showing many overlapping pulses due to limited resolution of the narrow band detector. The overlapping pulses does not get recorded by the analyzer resulting in a reduced count of pulses with time.

The PD distributions have multimodal characteristics [3] and show little change in their shape. However, with ageing the part involving larger magnitude pulses show a change in shape $[1,2]$. The change in the shape of larger magnitude pulses is exclusively studied with help of skewness parameter. The skewness value is positive at inception and becomes negative before the failure of the sample. The change in the skewness value is often to char- 

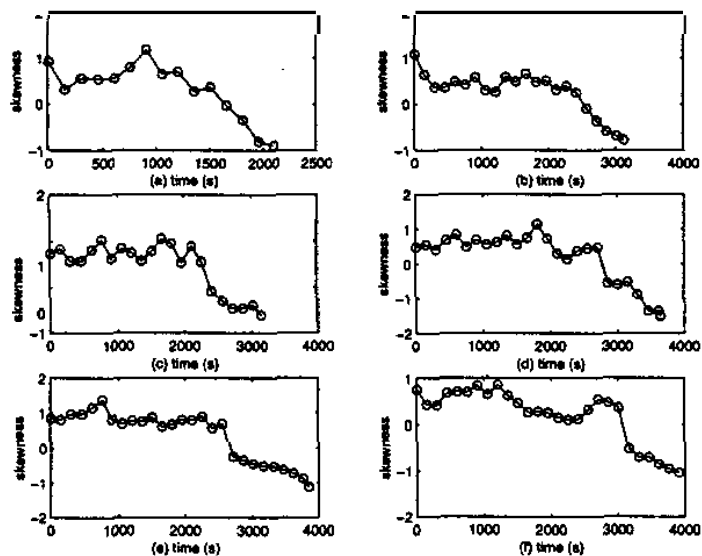

Figure 9: Skęrness value at different stages of ageing

acterize the state of insulation [4] and is therefore, computed at regular interval of time. Figure 9 gives the change in the skewness value with time for six samples. It can be seen from the figure that the skewness value at some point of time in the experiment becomes negative. The exact time of crossing the zero is obtained from the PD records. Table $\mathbf{1}$ shows the values of time to failure $(\mathrm{T})$, the time at which skewness became negative $(t)$ and the ratio of the two times $(t / T)$ for six samples. It is found that the ratio $(t / T)$ remains constant for all the samples even though the time to failure vary considerably. The observation of the constant ratio is useful to interpret the ageing phenomenon and for prediction of the remnant life of the sample by observing the instant of zero value of skewness.

Table 1: PD ageing characteristics

\begin{tabular}{|c|c|c|c|}
\hline $\begin{array}{c}\text { Sample } \\
\text { Number }\end{array}$ & $\begin{array}{c}\text { Time to } \\
\text { failure } \\
(\mathrm{T}) \mathrm{sec}\end{array}$ & $\begin{array}{c}\text { Skewness } \\
\text { crossover } \\
(\mathrm{t}) \mathrm{sec}\end{array}$ & $\begin{array}{c}\text { Ratio } \\
\left(\frac{t}{T}\right)\end{array}$ \\
\hline \hline 1. & 2100 & 1640 & 0.7809 \\
\hline 2. & 3120 & 2510 & 0.8044 \\
\hline 3. & 3130 & 2440 & 0.7795 \\
\hline 4. & 3640 & 2840 & 0.7802 \\
\hline 5. & 3850 & 3050 & 0.7922 \\
\hline 6. & 3910 & 3060 & 0.7826 \\
\hline
\end{tabular}

\section{Conclusion}

The paper is concluded with the following comments:
1. The PD detector-analyzer measure only a part of the PD activity determined by its limits. For meaningful interpretation of PD distributions a close attention to these limits is required.

2. The PD detector limit is used to advantage for ageing characterization. The paper demonstrate the use of skewness parameter for higher mode of PD distribution.

3. The zero crossover point of skewness value is characteristic and useful for diagnosis.

\section{References}

[1] S. Senthil Kumar, Y.P. Nerkar, M.N. Narayanachar and R.S. Nema, "Influence of detectors on measurement of partial discharges", $12^{\text {th }}$ International Symposium on High Voltage Engineering, Bangalore, India, 6-661047-1050, August 2001:

[2] Y.P. Nerkar, S. Senthil Kumar, M.N. Narayanachar and R.S.Nema, "Partial Discharge Oscillograms and Pulse Distribution in Transformer Oil-Pressboard Insulation", International Conference on Dielectric Liquid, Nara, Japan, 20-25 July,1999.

[3] Y.P. Nerkar, M.N. Narayanachar and R.S.Nema, "Characterisation of partial discharges in oil impregnated pressboard insulation systems", $11^{\text {th }}$ International Symposium on High Voltage Engineering, London, UK, 5.208.P5-5.211.P5, August 1999.

[4] T. Okamoto and T. Tanaka, "Novel partial discharge computer-aided measurement systems", IEEE Trans. Elec. Instu,, Vol-21, pp-10151019, 1986.

Author's addresses:

Department of High Voltage Engineering

Indian Institute of Science

Bangalore, INDIA- 560012

Emails:

senthil@hve.iisc.ernet.in

nerkar@hve.iisc.ernet.in

mnn@hve.iisc.ernet.in

rsnema@hve.iisc.ernet.in 